\title{
Ancestral cells in two-dimensional soap froth
}

\author{
W. Y. Tam, K. M. Cheung, and K. Y. Szeto \\ Department of Physics, The Hong Kong University of Science and Technology, Clear Water Bay, Kowloon, Hong Kong
}

(Received 1 July 1997)

\begin{abstract}
From the evolution of the ancestral cells in two-dimensional soap froth, we find that the ancestral cell mean area $\left\langle A_{a n}\right\rangle$ decreases and the mean number of edges $\left\langle n_{a n}\right\rangle$ increases nonlinearly backwards in time. Unlike surviving cells, there is no sign of fixed point for the ancestral cells in our experiment. Also, the normalized mean area $\left\langle A_{a n}(t)\right\rangle /\left\langle A_{a n}\left(t_{f}\right)\right\rangle$ and mean number of edges $\left\langle n_{a n}(t)\right\rangle$ form scaling functions independent of $t_{f}$ $>t$ with the normalized mean area $\langle A(t)\rangle /\left\langle A\left(t_{f}\right)\right\rangle$ of the whole froth. These results agree with a dynamical simulation for two-dimensional soap froths.
\end{abstract}

[S1063-651X(98)15206-6]

PACS number(s): 82.70.Rr, 02.50.-r, 05.70.Ln

Cellular structures such as metal grains and biological tissues are common in nature [1]. Among these systems, soap froth is particularly interesting for the study of the evolution of patterns because of the simplicity and relative ease of its experimental setup [2-5]. More importantly, the soap froth is a nonequilibrium system driven by gas diffusion that evolves to a universal scaling state widely observed in other nonequilibrium cellular systems. The topological properties of soap froths have now been well studied experimentally and good agreements with theories and simulations have been reported [5-8]. However, the study of the dynamics has been limited despite the confirmation of the linear growth rate for the mean bubble area and the dynamical scaling laws $[1,7,9]$. Currently, soap froths have again attracted much interest due to recent studies of surviving cells (survivors) [10-12] and first-passage exponent [13-15] in twodimensional soap froths. Studies of survivors have claimed the existence of a fixed point characterized by a stationary distribution of numbers of edges for the survivors [10-12]. The existence of a fixed point for survivors is nonintuitive and deserves detailed confirmation [16]. In this paper, instead of tracking the survivors, we measure the properties of ancestral cells (ancestors) of the bubbles taken at final time $t_{f}$ by following them backwards in time within the scaling state of the froth. We find that the normalized mean area and mean number of edges of the ancestors form scaling functions of the normalized mean area for the whole froth, independent of the final time $t_{f}$. More importantly, the data do not seem to reach any fixed point as observed previously in the studies of survivors [10-12]. We also have performed dynamical simulations for the two-dimensional soap froth and the results agree well with the experiment, suggesting that the fixed point may be reached only after a very long time if it exists at all.

We first clarify the difference between the survivors and ancestors. In previous studies [10-12], survivors were defined as bubbles chosen from an initial state in the scaling regime at time $t_{0}$ such that they survive until time $t>t_{0}$. As $t$ is increased from $t_{0}$ to the final time $t_{f}$, different bubbles will be chosen as the survivors but they are all subsets of the initial state. Thus, survivors are statics. Here, ancestors are defined as the predecessors of the bubbles at the final times $t_{f}$ and are chosen from states at various times $t<t_{f}$. At a particular time $t<t_{f}$, subset $\left\{\mathcal{S}_{a n}\left(t, t_{f}\right)\right\}$ of the froths $\{\mathcal{S}(t)\}$ at $t$ can be identified as the ancestors of the bubbles $\left\{\mathcal{S}\left(t_{f}\right)\right\}$ belonging to the froth at $t_{f}$. In general, the number of edges of these ancestors $\left\{\mathcal{S}_{a n}\left(t, t_{f}\right)\right\}$ can be different from the bubbles (a)

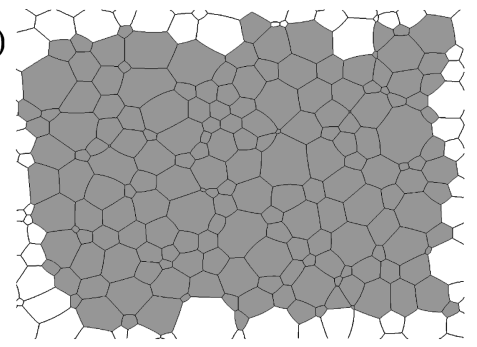

(c)

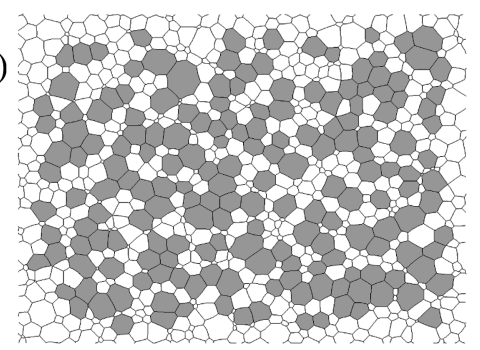

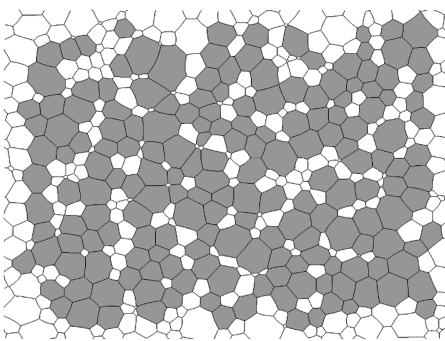

(b)

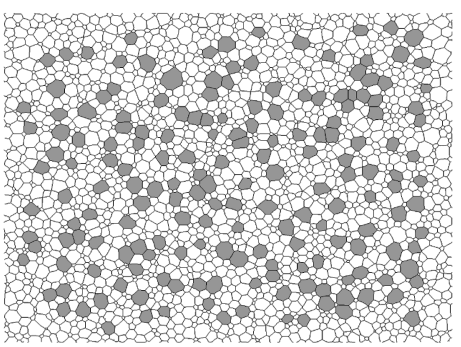

FIG. 1. Images of soap froth taken at time (a) $150.0 \mathrm{~h}$, (b) $61.17 \mathrm{~h}$, (c) $29.67 \mathrm{~h}$, and (d) $0.0 \mathrm{~h}$. The shaded cells are ancestors starting with $t_{f}=$ $150.0 \mathrm{~h}$ backward in time. 

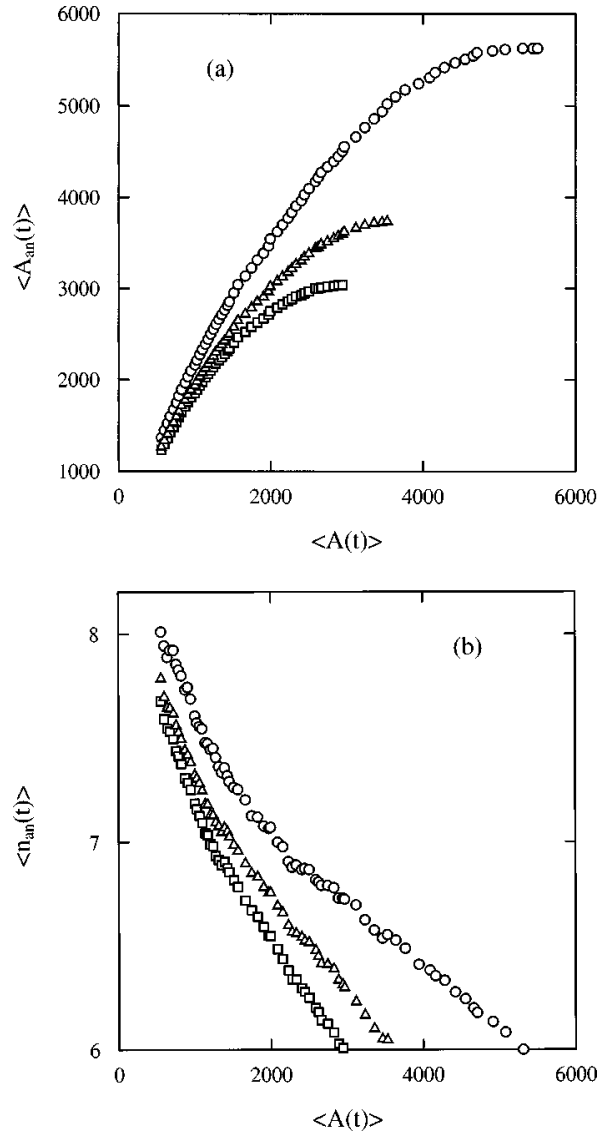

FIG. 2. (a) Mean area of the ancestors $\left\langle A_{a n}(t)\right\rangle$ vs the mean area of the whole froth $\langle A(t)\rangle$ for $t_{f}=$ (open circles) $150.0 \mathrm{~h}$, (open triangles) $92.76 \mathrm{~h}$, and (open squares) $70.5 \mathrm{~h}$. (b) Mean number of edges of the ancestors $\left\langle n_{a n}(t)\right\rangle$ vs the mean area of the whole froth $\langle A(t)\rangle$ for $t_{f}=$ (open circles) $150.0 \mathrm{~h}$, (open triangles) $92.76 \mathrm{~h}$, and (open squares) $70.5 \mathrm{~h}$.

in $\left\{\mathcal{S}\left(t_{f}\right)\right\}$. The concept of ancestors can be generalized beyond the scaling regime to the theoretical regime with the origin of time defined by the extrapolation of the linear time dependence of mean area to zero area. Therefore, one can treat the ancestors as primordial bubbles that exist since the origin of time. The evolution of the ancestors thus maps out the history of the bubbles from primordial time, where there are infinitely many bubbles with vanishing area so that the total area corresponds to the area of the sample. The dynamics and topological properties of these ancestors are very different from those of the survivors which have no natural time origin, but have the natural end of time at $t=\infty$.

Our experimental setup has been reported recently in a study of the first-passage exponent of the unswept area in two-dimensional soap froths [15]. The experiment started with about 20000 bubbles and had about 6000 in the scaling state, determined by monitoring the stationarity of the distribution of the number of edges. The experiment lasted for one week, with 197 bubbles at the end. A high-resolution chargecoupled-device digital camera $(1037 \times 1344$ pixels $)$ was used to capture images of the froth every $10 \mathrm{~min}$ during its evolution. The ancestors of the bubbles taken at a late stage of the froth were identified by following the bubbles frame by frame backwards in time. This was done automatically by a computer for most of the bubbles and manually for a few that
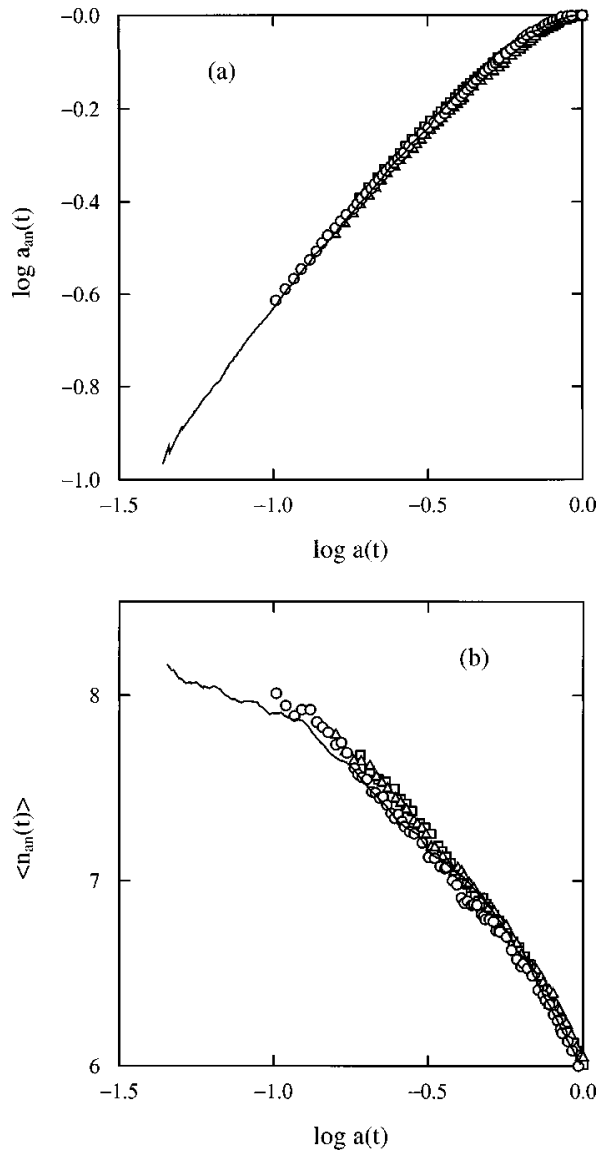

FIG. 3. (a) Log-log plot of the normalized mean area of the ancestors $a_{a n}(t)$ vs the normalized mean area of the whole froth $a(t)$ for $t_{f}=$ (open circles) $150.0 \mathrm{~h}$, (open triangles) $92.76 \mathrm{~h}$, and (open squares) $70.5 \mathrm{~h}$. (b) Mean number of edges of the ancestors $\left\langle n_{a n}(t)\right\rangle$ vs the log of the normalized mean area of the whole froth $a(t)$ for $t_{f}=$ (open circles) $150.0 \mathrm{~h}$, (open triangles) $92.76 \mathrm{~h}$, and (open squares) $70.5 \mathrm{~h}$.

could not be identified by the computer. Bubbles that wandered in and out of the image frame during the course of the evolution were also excluded.

Figure 1 shows the ancestor evolution backwards in time starting from the latest time $t_{f}=150.0 \mathrm{~h}$ (a) with 181 (surviving) bubbles to time (b) $61.17 \mathrm{~h}$, (c) $29.67 \mathrm{~h}$, and (d) $0.0 \mathrm{~h}$, where there are 2135 bubbles for the whole froth. The shaded cells are the 181 ancestors of the surviving bubbles at time $t_{f}=150.0 \mathrm{~h}$. The temporal dependence of the mean area $\left\langle A_{a n}(t)\right\rangle$ and the mean number of edges $\left\langle n_{a n}(t)\right\rangle$ of these ancestors are shown as open circles in Figs. 2(a) and 2(b), respectively. Here, we choose to use the mean area $\langle A(t)\rangle$ of the whole froth as the time scale, since $\langle A(t)\rangle$ has linear time dependence in the scaling state. Also shown in Fig. 2 are results obtained using $t_{f}=92.76 \mathrm{~h}$ (open triangles) and $t_{f}=$ $70.50 \mathrm{~h}$ (open squares) with 280 and 354 surviving bubbles, respectively. All data sets show similar behaviors with $\left\langle A_{a n}(t)\right\rangle$ decreasing and $\left\langle n_{a n}(t)\right\rangle$ increasing nonlinearly backwards in time. We find that both $\left\langle A_{a n}(t)\right\rangle$ and $\left\langle n_{a n}(t)\right\rangle$ form scaling functions as shown in Fig. 3 when rescaled. The normalized mean area of ancestors $a_{a n}(t)$ $=\left\langle A_{a n}(t)\right\rangle /\left\langle A_{a n}\left(t_{f}\right)\right\rangle$ is plotted, in logarithmic scales, against the normalized mean area of the whole froth $a(t)$ $=\langle A(t)\rangle /\left\langle A\left(t_{f}\right)\right\rangle$ for the three $t_{f}$ 's in Fig. 2(a). Data collapse 


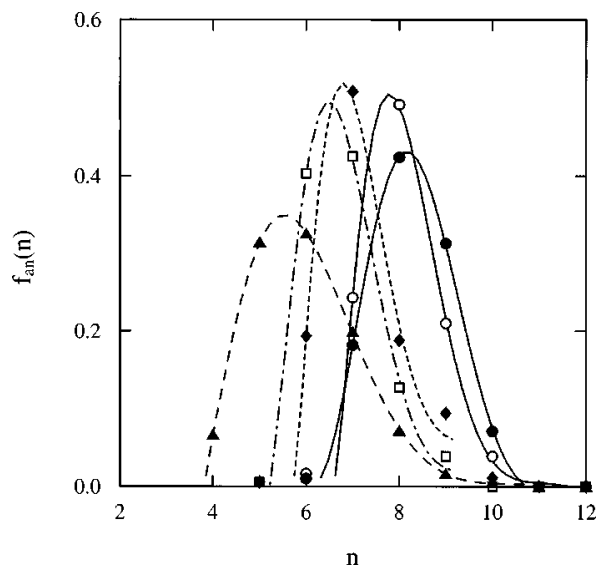

FIG. 4. Distribution of the number of edges for the ancestors as shown in Fig. 1. The corresponding symbols for Figs. 1(a), 1(b), $1(\mathrm{c})$, and 1(d) are solid triangles, open squares, solid diamonds, and open circles, respectively. Also shown is the distribution at the earliest time obtained from the dynamical simulation (solid circles). The lines are guides to the eyes.

is excellent. Good data collapse is also observed for the mean number of edges of the ancestors when plotted against the normalized mean area of the whole froth as shown in Fig. 3(b). It is seen in Fig. 3(a) that $a_{a n}(t)$ decreases more slowly backwards in time than $a(t)$, resulting in $a_{a n}(t) / a(t)$ larger than one for all times in the past. Furthermore, the dependence of $a_{a n}(t)$ upon $a(t)$ as shown in Fig. 3(a) can be approximated by a power law for a long time into the past. The exponent for the power law is estimated to be 0.83 using the last portion of the longest data set. However, this value is expected to increase for longer data sets. It can be seen from Fig. 3(b) that $\left\langle n_{a n}(t)\right\rangle$ increases from 6.0 at $t_{f}$ to 8.0 for the earliest time. Furthermore, it does not seem to reach a stationary value as one goes backward in time. This is supported by the distribution function of the number of edges for the ancestors $f_{a n}(n)$ as shown in Fig. 4 for the images in Fig. 1. It is clear that the distribution shifts continuously to higher $n$ as one goes further into the past. The distribution at the earliest state is similar to previous results with survivors [10-12] except that ours has a narrower distribution range. The results indicate that only bubbles with many edges and large areas are likely to survive long.

We use a recent dynamical model to simulate the twodimensional soap froth [17]. This model is quite efficient and has been shown to produce the correct dynamics for twodimensional froths [18]. The simulation started with 5000 bubbles and was stopped when there were about 100 bubbles. The ancestors of the bubbles starting at different times $t_{f}$ were followed backwards in time in the scaling state to the earliest time with about 2000-3000 bubbles. Details of the simulation can be found in Refs. $[17,18]$, and results will be reported in another paper on survivors [16].

The solid lines in Figs. 3(a) and 3(b) are, averaged over five runs from the simulation, the normalized mean area $a_{a n}(t)$ and the mean number of edges $\left\langle n_{a n}(t)\right\rangle$ of the ancestors plotted as a function of the normalized mean area $a(t)$ for the whole froth. The agreement with experimental results is excellent. A power-law fit for $a_{a n}(t)$ versus $a(t)$ gives an exponent of $0.91 \pm 0.02$, slightly larger than that of the ex- periment, using data points with $a(t)$ less than 0.1 . It is also clear from Fig. 3(b) that $\left\langle n_{a n}(t)\right\rangle$ still increases with decreasing $a(t)$, indicating that the simulations have not reached the stationary fixed point as reported for the survivors. This is further supported by the continuous shifting of the distribution of $n_{a n}(t)$ to higher $n$, similarly to the experimental results. The distribution for the earliest time from the simulation is shown as solid circles in Fig. 4. The distribution has higher $n$ values than the experiment but is slightly "behind" the previous simulation $[11,12]$, due to the fact that the previous simulation had more bubbles and lasted longer.

From our data on ancestors, we find that there is a natural time scale for testing the existence of a fixed distribution $f_{a n}(n)$, namely, the time at the infinite past should have a vanishing point $\langle A(t=-\infty)\rangle$ where the number of bubbles is infinite. Although experimentally this is not achievable, this time scale is well defined and a measure from this time scale is given by the value of $\langle A(t)\rangle$. A question concerning a fixed distribution at infinite past is whether there is, first of all, the existence of a finite value of $n^{\star}$ such that it is the mean number of edges of the ancestors at infinite past. A second question is whether the variance of the ancestor distribution is finite or zero at infinite past. Our data indicates that the answer to the first question is likely to be negative. This is quite physical and intuitive, since the existence of a finite $n^{\star}$ for the ancestors in the infinite past implies that there is a special topological reason in the dynamics of soap froth evolution that fixes such a finite value of $n^{\star}$, and this is quite unlikely because the fundamental dynamics is von Neumann's law [1], which has only one special topological class: $n=6$, fixed by the Euler theorem in two-dimensional Euclidean space. Since $n^{\star}$ increases as we trace our ancestors backwards in time, the logical conclusion from our data is to say that $n^{\star}=\infty$. This is supported by a slightly negative exponent obtained for a power-law fit of $\left\langle n_{a n}(t)\right\rangle$ versus $a(t)$ for both experiment and simulation. Now, $n^{\star}=\infty$ corresponds to circular bubbles, implying that the only primordial ancestors that exist for all times are those with an infinite number of edges. The second question is more subtle, as it addresses the shape of the ancestor distribution. There are two scenarios: (1) the variance of the ancestor distribution decreases, but asymptotically to a finite value, or (2) the decreasing variance asymptotically vanishes. These two cases are difficult to test experimentally, but they correspond to two different possibilities with distinct physical implications. In the first case, the ancestor distributions are simply moving towards higher values of $n$ as one goes further into the past, but the shape of the distribution as measured by the variance is preserved. In the second case, the ancestor distributions converge to a $\delta$ distribution. At this point, our data cannot distinguish these two cases, but certainly an answer will give us more insights into the evolution of soap froth.

We acknowledge useful correspondence with B. Levitan. W. Y. Tam acknowledges support from the Direct Allocation Grant No. 96/97.SC22 of the Hong Kong University of Science and Technology. K. Y. Szeto acknowledges support from Hong Kong Telecom Institute of Information Technology. We thank J. J. Chae and M. Tabor for letting us use their dynamic code to simulate the soap froth evolution. 
[1] D. Weaire and N. Rivier, Contemp. Phys. 25, 59 (1984).

[2] J. A. Glazier, S. Gross, and J. Stavans, Phys. Rev. A 36, 306 (1987).

[3] J. A. Glazier, M. P. Anderson, and G. S. Grest, Phys. Mag. 62, 6115 (1990).

[4] E. A. Holm, J. A. Glazier, D. J. Srolovitz, and G. S. Grest, Phys. Rev. A 43, 2662 (1991).

[5] J. Stavans, Rep. Prog. Phys. 56, 733 (1993).

[6] H. Flyvbjerg, Phys. Rev. E 47, 4037 (1993); Physica A 194, 298 (1993).

[7] J. Stavans, E. Domany, and D. Mukamel, Europhys. Lett. 15, 479 (1991).

[8] T. Aste, K. Y. Szeto, and W. Y. Tam, Phys. Rev. E 54, 5482 (1996).

[9] K. Y. Szeto and W. Y. Tam, Physica A (to be published).
[10] B. Levitan, E. Slepyan, O. Krichevshy, J. Stavans, and E. Domany, Phys. Rev. Lett. 173, 756 (1994).

[11] B. Levitan and E. Domany, Phys. Rev. E 54, 2766 (1996).

[12] B. Levitan and E. Domany, Int. J. Mod. Phys. B 10, 3765 (1996).

[13] B. Derrida, V. Hakim, and V. Pasquier, Phys. Rev. Lett. 75, 751 (1995); B. Derrida, V. Hakim, and R. Zeitak, ibid. 77, 2871 (1996).

[14] S. N. Majumdar, C. Sire, A. J. Bray, and S. Cornell, Phys. Rev. Lett. 77, 2867 (1996).

[15] W. Y. Tam, R. Zeitak, K. Y. Szeto, and J. Stavans, Phys. Rev. Lett. 78, 1588 (1997).

[16] W. Y. Tam and K. Y. Szeto (unpublished).

[17] J. J. Chae, Ph.D. thesis, University of Arizona, 1995.

[18] J. J. Chae and M. Tabor, Phys. Rev. E 55, 598 (1996). 